\title{
Microemulsion Polymerization of Butyl Acrylate. Effect of Radical Scavenger and Formation of Primary Radicals
}

\author{
Ignác $\mathrm{CAPEK}^{\dagger}$ and Viera JURANICǑvá \\ Polymer Institute, Slovak Academy of Sciences, 84236 Bratislava, \\ Dúbravská cesta 9 , Slovakia
}

(Received October 19, 1998)

\begin{abstract}
Microemulsion polymerizations of butyl acrylate initiated by dibenzoyl peroxide in the presence of a radical scavenger (RS)\{stable radicals : an oil soluble RS : 4-stearoyloxy-2,2,6,6-tetramethyl piperidine-1-oxyl (STPO) and water soluble RS : 4-hydroxy-2,2,6,6-tetramethyl piperidine-1-oxyl (HTPO)|were investigated. STPO and HTPO decreased the rate of polymerization and the decrease was more pronounced with STPO. Inorganic salts and organic additives decreased the rate of polymerization. The shift of the maximum rate of polymerization to lower conversion by increasing temperature and addition of several additives was attributed to increase of particle number and decrease of monomer concentration at the reaction loci. The decrease of the particle size in the presence of RS is attributed to retardation of particle growth. A close packed structure at the droplet surface promoted by current additives acts as a barrier for entering radicals. The larger overall activation energy in runs with STPO is discussed in terms of a depressed entry of radicals into microdroplets.

KEY WORDS Microemulsion / Butyl Acrylate / Polymerization / Tetramethyl Piperidine-1-oxyl Radical Scavenger / Inorganic Salts / Rate of Polymerization / Polymer Particles /
\end{abstract}

In the case of microemulsion polymerization initiated by the oil-soluble initiator, there are two main approaches for the production of primary radicals $:^{1-3} 1$ ) in the monomer swollen micelles or polymer particles radicals desorb to the aqueous phase and 2) in the aqueous phase radicals are generated from the fraction of the oilsoluble initiator dissolved in water. ${ }^{4-6} \mathrm{~A}$ partly watersoluble initiator such as $\alpha, \alpha^{\prime}$-azobis(isobutyronitrile) (AIBN) forms primary radicals in both the aqueous phase and in the monomer-swollen micelles. In the case of highly hydrophobic initiator the formation of radicals from the fraction of initiator dissolved in the aqueous phase is negligible, as well as exit of hydrophobic primary radicals into the aqueous phase. Microemulsion and miniemulsion polymerizations initiated by hydrophobic initiators such as dibenzoyl peroxide (DBP) or lauroyl peroxide (LPO) are reported to be relatively fast. ${ }^{1,2,7}$ The very low water solubility of hydrophobic initiator (DBP, LPO, etc.) and radical fragments propose a very low rate of polymerization ( $c a$. by two orders in magnitude lower than that for peroxodisulfate initiated polymerization). The strong cage effect within the microdroplets or latex particles depresses the formation of radicals in the monomer phase. However, the rate of DBP-initiated polymerization was $c a$. 5 times smaller than the water-soluble peroxodisulfate initiated polymerization (polymerizations carried out under the same reaction conditions). Thus, the polymerization behavior is not properly described by two reaction approaches : 1 ) water-phase initiation and 2) desorption of primary (monomeric) radicals formed in the microdroplets in the aqueous phase.

The non-linearity between the molecular weights of the polystyrene (much below $10^{5}$ ) and laser repetition rate was attributed to the droplet and particle collisions. ${ }^{8}$ In the microemulsion polymerization of styrene

\footnotetext{
${ }^{\dagger}$ To whom all correspondence should be addressed.
}

initiated by pulsed UV light (with AIBN), polymer particles grew via supply of monomer from the microemulsion droplets (and inactive polymer particles) by diffusion or collisions. Collisions of polymer particles and microdroplets were also proved by Kim and Napper. ${ }^{9}$ The latter, may thus be a further process which co-operates with above two ones in generation of initiating radicals. For example, pseudo-bulk kinetics operative for the oilsoluble initiator, 2, 2'-azobis-(2-methyl butyronitrile) (AMBN) may explain why the molecular weight dependence on initiator concentration is much higher for $\operatorname{AMBN}(-0.55$ power $)$ than for pottassium peroxadisulfate (KPS) $(-0.07) .^{10}$

In microemulsion systems, collisions lead to the transfer of the reactants including hydrophobic initiator between droplets or within transient droplet clusters (percolation). The decay of droplet clusters promotes the exit of radicals from radical pairs (a cage effect) into individual droplets. This might be the reason why the hydrophobic initiator is very efficient in the production of primary radicals in the microemulsion polymerization. A possible way of primary (initiating) radical formation was followed using an inhibitor or retarder approach. ${ }^{11-16}$ We suppose that the location of RS in different phases varies the rate of radical deactivation and the flux of radicals. Modification of droplet surface caused by the presence of additives is supposed to vary the radical entry rate and the transfer of radicals between the droplets. This was why we investigated the effects of RS and several additives on the kinetic and colloidal parameters of the microemulsion polymerization of butyl acrylate (BA) initiated by the oil-soluble initiator DBP.

\section{EXPERIMENTAL}

\section{Materials}

Commercially available butyl acrylate (BA) was purified by the usual methods. ${ }^{11,17}$ Extra pure dibenzoyl per- 
oxide (DBP, Fluka) was used as supplied. The emulsifier used was the reagent-grade sodium dodecylsulfate (SDS, Fluka). Stable nitroxyl radicals STPO (4-stearoyloxy2,2,6,6-tetramethyl piperidine-1-oxyl, oil-soluble) and HTPO (4-hydroxy-2,2,6,6-tetramethyl piperidine-1-oxyl, water-soluble) were used to inhibit growth either in the monomer or aqueous phase. Inorganic salts $\left(\mathrm{FeCl}_{2} \cdot 4\right.$ $\mathrm{H}_{2} \mathrm{O}, \mathrm{CuCl}_{2} \cdot 2 \mathrm{H}_{2} \mathrm{O}, \mathrm{KJ} \cdot 4 \mathrm{H}_{2} \mathrm{O}$ ) and organic additives (maleic anhydride (MAn) and benzoic acid (BAc)) used were of analytical-grade. Doubly distilled water was used as the polymerization medium.

\section{Polymerization Procedure}

Batch polymerizations were carried out at 60,70 , and $80^{\circ} \mathrm{C}$. In all runs the recipe was $100 \mathrm{~g}$ water, $10 \mathrm{~g} \mathrm{BA}, 20$ g SDS, $0.025 \mathrm{~g} \mathrm{NaHCO}, 1 \times 10^{-3} \mathrm{~mol} \mathrm{dm}^{-3} \mathrm{DBP}, 5 \times$ $10^{-5} \mathrm{~mol} \mathrm{dm}{ }^{-3}$ STPO or HTPO, and $1 \times 10^{-3} \mathrm{~mol} \mathrm{dm}^{-3}$ $\mathrm{FeCl}_{2} \cdot 4 \mathrm{H}_{2} \mathrm{O}, \mathrm{CuCl}_{2} \cdot 2 \mathrm{H}_{2} \mathrm{O}, \mathrm{KJ} \cdot 4 \mathrm{H}_{2} \mathrm{O}, \mathrm{MAn}$ or BAc. Conversion of monomer was determined by dilatometric measurements (checked by gravimetry). The rate of polymerization $\left(R_{\mathrm{p}}=(\mathrm{d} x / \mathrm{d} t) n_{\mathrm{M}}\right)$ is estimated from the conversion-time dependence $(\mathrm{d} x / \mathrm{d} t$, where $x$ is the weight fraction of monomer to polymer) and the initial number of moles of monomer $\left(n_{\mathrm{M}}\right)$ present per unit volume of the aqueous phase. ${ }^{4}$

\section{Polymer and Latex Characterization}

Polymerization and measurement of particle $\operatorname{size}\left(D_{\mathrm{n}}\right)$ and number $\left(N_{\mathrm{p}}\right)$ (by light scattering) were the same as described earlier. ${ }^{2,13}$ Particle size distribution(PSD) was estimated as described by Schork et al.$^{18}$ Limiting viscosity numbers $[\eta]$ were determined with Ubbelohde viscometer in acetone at $25^{\circ} \mathrm{C}$ and used to estimate viscosity-average molecular weights. ${ }^{3,19}$

\section{RESULTS AND DISCUSSION}

\section{Polymerization Rate}

Conversion-time data for the microemulsion polymerization of BA initiated by the oil-soluble DBP initiator are shown in Figures 1 and 2. In these runs, S-shaped conversion curves and final conversion close to $100 \%$ conversion were achieved. In the presence of HTPO/KJ, $\mathrm{FeCl}_{2}, \mathrm{KJ}$, and $\mathrm{BAc}$ final conversion is much below $100 \%$ (Table I ). This is attributed to particle agglomeration. Consumption of initiator via the redox reaction is not ruled out.

The distribution coefficient for DBP (BA/water, 1/1(v/ v) was estimated to be $99 / 1$ to $98 / 2$ (weight ratio).This indicates that the water-phase initiation should be negligible due to the fact that DBP is mainly located in the microdroplets. However, the polymerization can only start when one radical from the radical pair (located in the microdroplet) exits to the aqueous phase or enters the second droplet. Therefore, the aqueous phase saturated with desorbed radicals is a part of the whole multiphase reaction system. The addition of $\mathrm{RS}$ retards (or inhibits) the polymerization either in the aqueous phase or monomer phase or in both phases.

Table II shows that colloidal and kinetic parameters of microemulsion polymerization to vary with increasing temperature and type of RS. The inhibition period decreases with increasing temperature. The rate of polym-

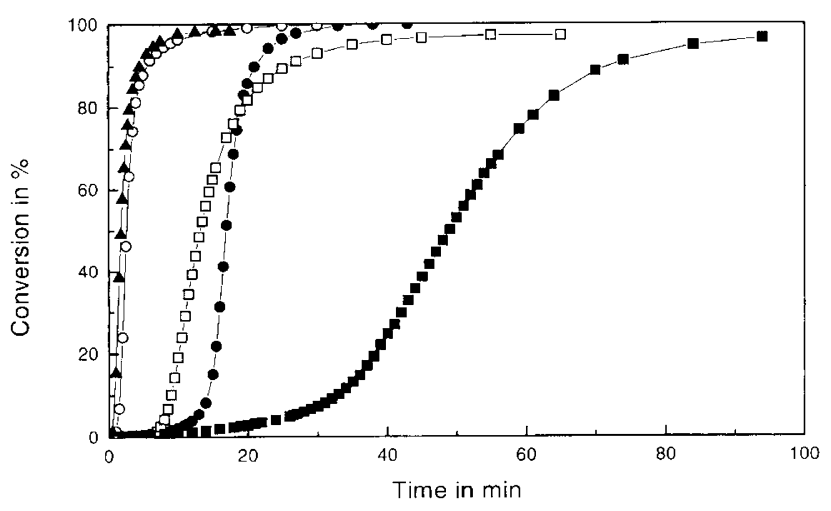

Figure 1. Variation of monomer conversion in the microemulsion polymerization of BA with reaction time and additive. Recipe, cf. EXPERIMENTAL. [DBP] $=1.0 \times 10^{-3} \mathrm{~mol} \mathrm{dm}^{-3} ;(\boldsymbol{\Delta})$ : without RS and additives, $(O)$ : [STPO] $=5 \times 10^{-5} \mathrm{~mol} \mathrm{dm}^{-3}$ and $\left[\mathrm{CuCl}_{2}\right]=1 \times$ $10^{-3} \mathrm{~mol} \mathrm{dm}^{-3},(\square):[\mathrm{STPO}]=5 \times 10^{-5} \mathrm{~mol} \mathrm{dm}^{-3}$ and $[\mathrm{BAc}]=1 \times$ $10^{-3} \mathrm{~mol} \mathrm{dm}{ }^{3},(\bigcirc):[\mathrm{HTPO}]=5 \times 10^{-5} \mathrm{~mol} \mathrm{dm}^{-3}$ and $\left[\mathrm{CuCl}_{2}\right]=1$ $\times 10^{-3} \mathrm{~mol} \mathrm{dm}^{-3},(\square)[\mathrm{HTPO}]=5 \times 10^{-5} \mathrm{~mol} \mathrm{dm}^{-3}$ and $[\mathrm{BAc}]=1 \times$ $10^{-3} \mathrm{~mol} \mathrm{dm}^{-3}$.

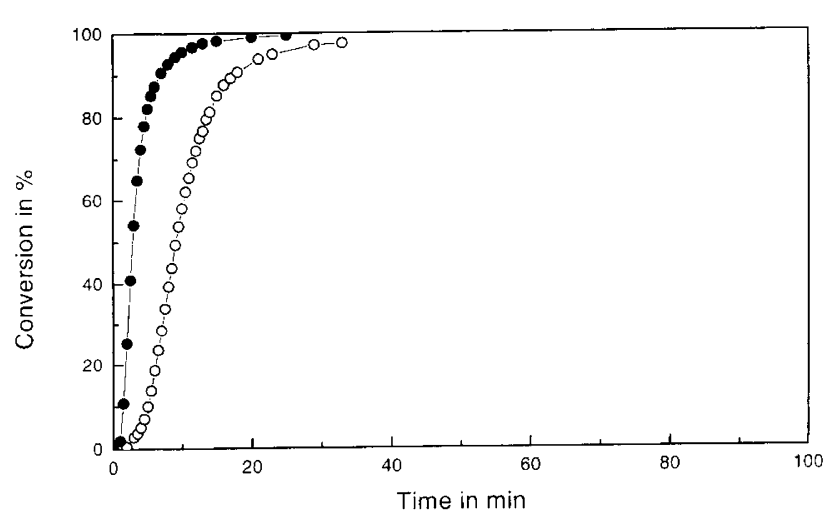

Figure 2. Variation of monomer conversion in the microemulsion polymerization of BA with reaction time and RS. Recipe, $c f$. EXPERIMENTAL. [DBP] $=1.0 \times 10^{-3} \mathrm{~mol} \mathrm{dm}^{-3} ;(\mathrm{O}):[\mathrm{HTPO}]=5 \times$ $10^{-5} \mathrm{~mol} \mathrm{dm}^{-3}(\bigcirc):$ [STPO] $=5 \times 10^{-5} \mathrm{~mol} \mathrm{dm}^{-3}$.

erization increases with temperature. This is attributed to increase of the rate of initiation (the increased level of primary radicals formed by decomposition of initiator) and the rate of particle formation.

Maximum rates of polymerization $\left(R_{\mathrm{p}, \max }\right)$ were used to get Arrenius plots (ln $R_{\mathrm{p}, \max }$ vs. 1/T) and overall activation energy $\left(E_{0}\right)$ as well. $E_{\mathrm{o}}$ was found to be $107 \mathrm{~kJ}$ $\mathrm{mol}^{-1}$ for STPO and $83 \mathrm{~kJ} \mathrm{~mol}^{-1}$ for HTPO, respectively. The higher inhibition efficiency of RS, the larger overall activation energy. STPO acts as a surface-active component with critical micellar concentration $\mathrm{ca} .4 \times 10^{-7} \mathrm{~mol}$ $\mathrm{dm}^{-3} \cdot{ }^{20}$ The water solubility of STPO is below $10^{-6} \mathrm{~mol}$ $\mathrm{dm}^{-3}$. ESR study of microemulsions saturated by RS showed that the radical group of STPO is located in a nonpolar environment. ${ }^{21}$ The (co)emulsifier properties of STPO propose that STPO penetrates the interface layer and forms a complex with the emulsifier. ${ }^{22}$ This interaction increases the residence time of STPO in microdroplets. The STPO/SDS complex produces a barrier for exiting and entering radicals. This increases the activation energy of initiation. In the first step the radical escapes from the cage (the microdriplet). In the second step (sin- 
Table I. Variation of kinetic, molecular weight and colloidal parameters in the microemulsion polymerization of BA with additive ${ }^{\mathrm{a}}$

\begin{tabular}{|c|c|c|c|c|c|c|c|c|}
\hline Additives & $\frac{R_{\mathrm{p}, \max } 10^{3^{\mathrm{b}}}}{\mathrm{mol} \mathrm{dm}^{-3} \mathrm{~s}^{-1}}$ & \multicolumn{2}{|c|}{$\frac{\text { Conv. } R_{\mathrm{p}, \mathrm{max}}{ }^{\mathrm{c}}}{\%}$} & \multirow{2}{*}{$\frac{\frac{\mathrm{Mv} 10^{6 \mathrm{~d}}}{\%}}{98.3}$} & \multirow{2}{*}{$\frac{\frac{\text { Conv.f }^{\mathrm{e}}}{\mathrm{nm}}}{(\mathrm{a})}$} & \multirow{2}{*}{$\begin{array}{l}\frac{D_{\mathrm{n}}{ }^{\mathrm{f}}}{\mathrm{dm}^{3}} \\
(\mathrm{~b}) \\
45\end{array}$} & \multirow{2}{*}{$\begin{array}{c}N_{\mathrm{p}} 10^{\cdot 18 \mathrm{~g}} \\
\frac{(\mathrm{a})}{2.7}\end{array}$} & \multirow{2}{*}{$\begin{array}{l}\mathrm{PSD}^{\mathrm{t}} \\
\frac{(\mathrm{a})}{1.33}\end{array}$} \\
\hline None $^{\mathrm{i}}$ & 6.0 & 32 & 1.5 & & & & & \\
\hline STPO & 1.4 & 30 & 0.69 & 97.5 & 30 & 35 & 6.3 & 1.45 \\
\hline $\mathrm{STPO} / \mathrm{CuCl}_{2}$ & 2.62 & 40 & 0.836 & 100 & 34 & 39 & 4.4 & 1.35 \\
\hline $\mathrm{STPO} / \mathrm{FeCl}_{2}$ & $2.5^{j}$ & - & 0.61 & 100 & 34 & 36 & 4.4 & 1.35 \\
\hline STPO/KJ & 1.35 & 10 & 0.92 & 92.5 & 90 & 42 & 0.04 & 1.57 \\
\hline STPO/MAn & 1.24 & 10 & 0.69 & 100 & 30 & 33 & 6.4 & 1.43 \\
\hline STPO/BAc & 0.4 & $20-60$ & 0.7 & 96.4 & 34 & 35 & 4.2 & 1.37 \\
\hline HTPO & 3.9 & 20 & 0.91 & 99.4 & 41 & 43 & 2.5 & 1.36 \\
\hline $\mathrm{HTPO} / \mathrm{CuCl}_{2}$ & 5.6 & 34 & 0.8 & 99.6 & 40 & 44 & 2.7 & 1.3 \\
\hline $\mathrm{HTPO} / \mathrm{FeCl}_{2}$ & 4.5 & 35 & 1.05 & 100 & 40 & 42 & 2.7 & 1.33 \\
\hline HTPO/KJ & $2.1^{\mathrm{j}}$ & - & 0.64 & 37.5 & 62 & 40 & 0.27 & 1.39 \\
\hline HTPO/BAc & 1.35 & 25 & 0.7 & 97 & 50 & 46 & 1.35 & 1.35 \\
\hline $\mathrm{CuCl}_{2}$ & 3.0 & 23 & 0.9 & 89.5 & 47 & 47 & 1.5 & 1.49 \\
\hline $\mathrm{FeCl}_{2}$ & $1.5^{j}$ & - & & & & & & \\
\hline $\mathrm{KJ}$ & $1.3^{\mathrm{j}}$ & - & 0.37 & & 313 & 47 & 0.006 & 1.47 \\
\hline MAn & 3.1 & 24 & 0.9 & 100 & 51 & 52 & 1.3 & 1.25 \\
\hline $\mathrm{BAc}$ & 3.0 & 23 & 0.97 & 37.5 & 52 & 52 & 0.6 & 1.36 \\
\hline
\end{tabular}

${ }^{\mathrm{a}}[\mathrm{DBP}]=1.0 \times 10^{-3} \mathrm{~mol} \mathrm{dm}{ }^{-3},[\mathrm{STPO}]=5 \times 10^{-5} \mathrm{~mol} \mathrm{dm}{ }^{-3}$, and temp $80^{\circ} \mathrm{C} .{ }^{\mathrm{b}}$ The maximum rate of polymerization. ${ }^{\mathrm{c}} \mathrm{Conversion}$ at which the maximum rate appeared. ${ }^{\mathrm{d}}$ Viscosity-average molecular weight of final polymer. ${ }^{\mathrm{e}}$ Final conversion. ${ }^{\mathrm{f}}$ Particle size diameter. (a) aging time $=0$, (b) aging time $=1$ year. ${ }^{\mathrm{g}}$ Number of final polymer particles. ${ }^{\mathrm{h}}$ Particle size distribution. ${ }^{\mathrm{i}}$ Without STPO and additive. ${ }^{\mathrm{j}}$ Determined by gravimetry at ca. $20-50 \%$ conversion.

Table II. Variation of kinetic and colloidal parameters of microemulsion polymerization of BA in the presence of RS with temperature ${ }^{\mathrm{a}}$

\begin{tabular}{|c|c|c|c|c|c|c|c|c|c|}
\hline Additive & $\frac{\text { Temp }}{{ }^{\circ} \mathrm{C}}$ & 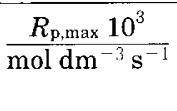 & \multicolumn{2}{|c|}{$\frac{\frac{\text { Conv. } R_{p}, \max }{\%}}{\frac{m_{0}}{\text { mol particle }{ }^{-1} \mathrm{~s}^{-1}}}$} & $\frac{R_{\mathrm{pp}} 10^{22 b}}{\min }$ & $\begin{array}{r}\mathrm{IP}^{\mathrm{c}} \\
\mathrm{nm} \\
(\mathrm{a})\end{array}$ & $\begin{array}{r}\frac{D_{\mathrm{n}}}{\mathrm{dm}^{3}} \\
\text { (b) }\end{array}$ & $\begin{array}{c}N_{\mathrm{p}} 10 \cdot 18 \\
\text { (a) }\end{array}$ & $\begin{array}{l}\text { PSD } \\
\text { (a) }\end{array}$ \\
\hline \multirow[t]{3}{*}{ STPO } & 60 & 0.16 & 50 & 0.36 & 80 & 34 & 42 & 4.4 & 1.46 \\
\hline & 70 & 0.5 & 40 & 1.0 & 57 & 32 & & 5.0 & \\
\hline & 80 & 1.4 & 30 & 2.31 & 7 & 30 & 35 & 6.1 & 1.51 \\
\hline \multirow[t]{3}{*}{ HТPO } & 60 & 0.73 & 30 & 4.3 & 50 & 47 & 48 & 1.7 & 1.29 \\
\hline & 70 & 1.6 & 25 & 8.0 & 38 & 44 & & 2.0 & \\
\hline & 80 & 3.9 & 20 & 15.6 & 5 & 41 & 43 & 2.5 & 1.32 \\
\hline
\end{tabular}

${ }^{\mathrm{a}}$ See legend to Table I . ${ }^{\mathrm{b}}$ Rate per particle. ${ }^{\mathrm{c}}$ Inhibition period.

gle) primary radicals start chain growth in microdroplets or aqueous phase. The desorbed radicals re-enter the microdroplets. The higher activation energy of initiation, the higher is the overall activation energy.

Both HTPO and STPO decrease the rate of polymerization but the decrease is less pronounced with HTPO. The distribution coefficient for HTPO was reported to be $19 / 1$ (water/oil) in the w/o micromulsion system. ${ }^{23}$ This indicates that the larger part of HTPO is located in the aqueous phase. Thus, HTPO mainly terminates radicals formed by the decomposition of the initiator dissolved in water and partly radicals formed in microdroplets. The main part of HTPO is supposed to be consumed by reaction with primary radicals (desorbed from microdroplets) dissolved in water. Stable nitroxyl radicals react with DBP to form the intermediate product which decomposes in the alkaline medium. ${ }^{24}$ The intermediate product DBP/HTPO decomposed within the alkaline emulsifier layer of particles and so free HTPO takes part in termination. Thus, the entry of radicals into microdroplets saturated with HTPO and transfer of mobile radicals within the droplet clusters are not hindered and so $E_{0}$ $\left(83 \mathrm{~kJ} \mathrm{~mol}^{-1}\right)$ is very close to the value $\left(85 \mathrm{~kJ} \mathrm{~mol}^{-1}\right.$, without $\mathrm{RS}$ ) found in the microemulsion polymerization of styrene and BA. ${ }^{1,25}$

Collisions (interactions) of microdroplets with flexible surface film are attractive. Under such conditions, the transient droplet clusters promote the transfer of material between droplets. Low-molecular weight coemulsifiers are known to form intermediate droplet clusters in microemulsion systems. ${ }^{26}$ Additives such as $\mathrm{CuCl}_{2}$, $\mathrm{FeCl}_{2}, \mathrm{KJ}, \mathrm{MAn}$, and BAc form complexes with SDS and so a denser droplet surface is formed. ${ }^{27-30}$ The rigid surface film depresses interactive collisions between droplets, the transfer of radicals between droplets (by hoping) and radical entry events. ${ }^{22}$

Additives (inorganic salt and organic component) alone or their mixtures with RS influence $R_{\mathrm{p}, \max }$ in a complex way (Table I ). The redox system $\mathrm{DBP} / \mathrm{FeCl}_{2}$ accelerates the decomposition of DBP and so increase in $R_{\mathrm{p}}$ should appear. ${ }^{31}$ However, the reverse is true, i.e., $R_{\mathrm{p}, \max }$ decreases. This indicates that the largest part of $\mathrm{FeCl}_{2}$ is somehow immobilized within the inorganic salt/SDS complex. The very slow polymerization with long nucleation period was also observed in the mixed RS/BAc systems. Here the inhibition efficiency was more pronounced with STPO. The slow polymerization is also found in systems with strong base-acid complexes SDS/ BAc and STPO/BAc, known to form close packed structures (or rigid surface film). ${ }^{32}$ The distribution coefficient for $\mathrm{BAc}\{(\mathrm{BA} /$ water, $1 / 1(\mathrm{v} / \mathrm{v}))$ estimated to be $88 / 12$ (the weight ratio)\} indicates that BAc prefers more the 
monomer (microdroplet) phase and so its reaction with growing radicals (chain transfer events) takes place. The predominant location of STPO and SDS in the interfacial layer promotes interactions with BAc. The main location of HTPO in the aqueous phase disfavors interactions between HPTO and BAc. A more dense surface layer is expected to be formed by MAn due to its high ability to form donor acceptor complexes with electronrich compounds. ${ }^{33}$ However, the effect of MAn is more complex while decrease of $R_{\mathrm{p}, \max }$ can also be attributed to accumulation of maleic acid (hydrolytic product of MAn). During 24 h MAn (maleic acid) was mostly transformed from the monomer to aqueous phase. Inorganic salts act as radical scavengers. This is a well-known reaction of KJ with peroxide. ${ }^{31}$ The anion $\mathrm{J}^{-}$strongly interacts with the water/droplet interface and so prevents reactants (radicals, ions, etc.) from entering the interfacial layer. ${ }^{34} \mathrm{~A}$ small nonpolarizable ion such as sodium or chloride does not influence the entry of reactants into the interfacial layer; a large polarizable ion such as iodide slows down this penetration.

The dependence of rate of polymerization $v s$. conversion in the microemulsion polymerization of BA initiated by $\mathrm{DBP}$ at $60^{\circ} \mathrm{C}$ is described by a curve with maximum at ca. $30 \%$ conversion (Figures 3 and 4 ). The same dependence (a curve with a maximum) was observed in the microemulsion polymerization of $\mathrm{BA}$ in the presence of HTPO and inorganic salts. In runs with STPO the rate of polymerization was observed to vary slightly from 15 to $50 \%$. This may be attributed to the retardation caused by STPO. $R_{\mathrm{p}, \max }$ shifted to the lower conversion by the addition of HTPO (Table I ). This results from a higher consumption rate of HTPO at low conversion (HTPO is dissolved in water).

The lower rate of polymerization in the presence of STPO (beyond the inhibition period) may be attributed to increased residence time of $\mathrm{RS}$ in microdroplets. STPO molecules act as coemulsifier and thus are predominantly located at the interface water/particle surface. ${ }^{14}$ The molecular weight and high hydrophobicity of STPO promote the formation of rigid surface film ${ }^{35}$ and suppress Ostwald ripening. ${ }^{15}$ In the former case, the transfer of hydrophobic components (initiator or its radicals) from monomer droplets to droplets or polymer particles within the droplets or particles clusters through the interface between droplets during collision is reduced and the release of primary radicals is depressed. In the latter case, the depressed monomer droplet degradation depresses the transfer of monomer and hydrophobe(STPO) to the reaction loci. Thus, both monomer and STPO are consumed by lower rates. Similar but less pronounced trend was observed with HTPO where hydrophilic RS molecules mainly accumulate in water and therefore their interactions with more hydrophobic additives are reduced.

The maximum rate shifts to lower conversion with increasing temperature (Table II). This may be discussed as follows : 1) increase of radicals concentration in the system, 2) polymer-emulsifier interaction, and 3) reduction of a critical micellar concentration(CMC), degree of counterion binding, and micelle aggregation number $\left(N_{\mathrm{a}}\right){ }^{36}$ With increasing temperature, the concentrations of radicals and particles increase. Thus, with increasing

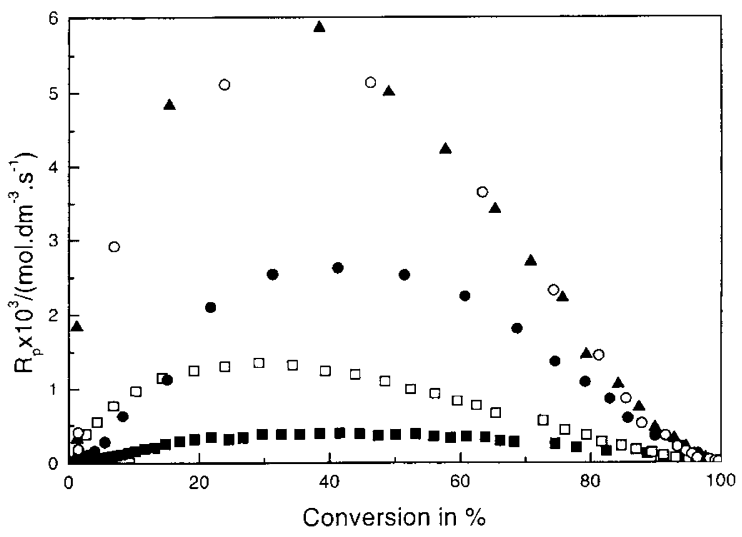

Figure 3. Variation of the rate of polymerization in the microemulsion polymerization of BA with conversion and additive. Recipe, cf. EXPERIMENTAL. $[\mathrm{DBP}]=1.0 \times 10^{-3} \mathrm{~mol} \mathrm{dm}-3,(\boldsymbol{A})$ : without RS and additives, $(O):\left[\right.$ STPO] $=5 \times 10^{-5} \mathrm{~mol} \mathrm{dm}^{-3}$ and $\left[\mathrm{CuCl}_{2}\right]=1 \times$ $10^{-3} \mathrm{~mol} \mathrm{dm}^{-3}$, ( $):[\mathrm{STPO}]=5 \times 10^{-5} \mathrm{~mol} \mathrm{dm}^{-3}$ and [BAc] $=1 \times$ $10^{-3} \mathrm{~mol} \mathrm{dm}^{-3}$, (O) : [HTPO] $=5 \times 10^{-5} \mathrm{~mol} \mathrm{dm}^{-3}$ and $\left[\mathrm{CuCl}_{2}\right]=1$ $\times 10^{-3} \mathrm{~mol} \mathrm{dm}^{-3},(\square)[\mathrm{HTPO}]=5 \times 10^{-5} \mathrm{~mol} \mathrm{dm}^{-3}$ and $[\mathrm{BAc}]=1 \times$ $10^{-3} \mathrm{~mol} \mathrm{dm}^{-3}$.

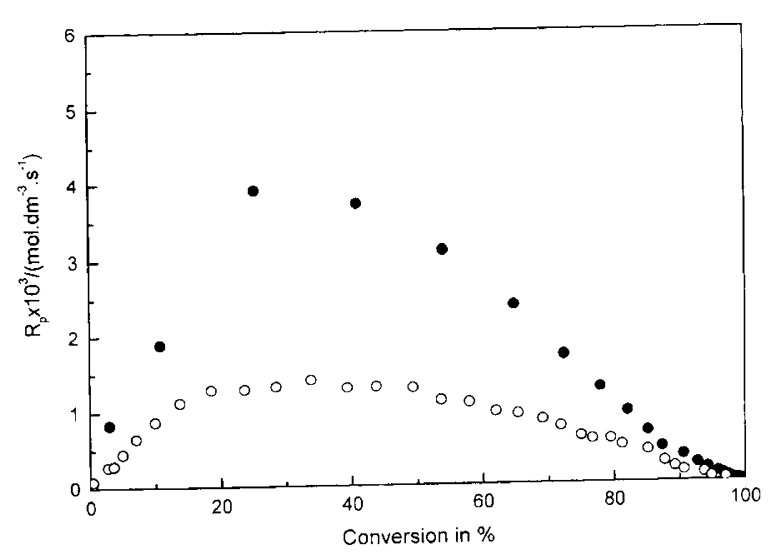

Figure 4. Variation of the rate of polymerization in the microemulsion polymerization of BA with conversion and RS. Recipe, $c f$. EXPERIMENTAL. [DBP] $=1.0 \times 10^{-3} \mathrm{~mol} \mathrm{dm}^{-3} ;(O):[\mathrm{HTPO}]=5 \times$ $10^{-5} \mathrm{~mol} \mathrm{dm}^{-3}(\bigcirc):\left[\right.$ STPO] $=5 \times 10^{-5} \mathrm{~mol} \mathrm{dm}^{-3}$

particle number the monomer cores of the microemulsion droplets disappear early in the polymerization and the monomer starved conditions become operative at lower conversion. The higher the particle concentration, the lower is the monomer concentration at the reaction loci. The interaction between polymer and emulsifier varies $N_{\mathrm{a}}$ and droplet number as well.

In the presence of an inorganic salt, adsorbed SDS is enhanced since repulsive force between the polar head groups of the emulsifier is reduced by (i) screening effects, (ii) lower degree of ionization of emulsifier molecules, and (iii) reduction in the hydration shell. ${ }^{37}$ Thus, a larger number of emulsifier molecules can be adsorbed on the droplet (particle) surface and packed more compactly at the surface with less energy. The dencer interface acts as a barrier to entering radicals. Cations can saturate the micellar surface or electrostatically neutralize the micelle surface charge and agglomeration of micelles or microdroplets occurs. ${ }^{38}$ 


\section{Colloidal Parameters}

Particle size decreases by the addition of STPO due to short-stopping of the growth events and thus the lifetime of active particles shortens. The accumulation of hydrophobic oligomers during the inhibition(nucleation) period (detected by GPC where $M_{w}$ varied $10^{3}-10^{4}$ ) disfavors the diffusional degradation of monomer microdroplets, transfer of monomer to the reaction loci and particle growth. ${ }^{39}$ The less pronounced particle decrease is observed in runs containing a STPO/inorganic salt $\left(\mathrm{CuCl}_{2}\right.$ and $\left.\mathrm{FeCl}_{2}\right)$ mixture. Inorganic salts $\left(\mathrm{CuCl}_{2} \cdot \mathrm{FeCl}_{2}\right.$, and $\mathrm{KJ}$ ), BAc and MAn alone were found to promote agglomeration and in some cases coagulum appeared. Inorganic salts promote irreversible association. ${ }^{40}$ The reverse is true for runs with $\mathrm{KJ}$ where particle size after 1 year aging strongly decreases. This can be attributed to partial coagulation of larger particles and disintegration of particle associates due to releasing $\mathrm{KJ}$ from particle clusters caused by oxidation of $\mathrm{KJ}$ by oxygen or initiator. ${ }^{31}$ Additives MAn and BAc promote the formation of stable polymer latexes; i.e., polymer latexes are stable after 1 year aging. Particle size is nearly independent of HTPO or HTPO/inorganic salt systems. HTPO molecules are mostly consumed during the inhibition period and therefore particle growth is similar to the system without RS. The addition of STPO increased PSD. Similar behavior was observed in runs with inorganic salts. The continual consumption of STPO leads to gradual increase of lifetime of active particle and particle size. Modification of droplet surface promotes agglomeration of latex particles and variation of PSD. The broader particle size distribution appears in runs with lower colloidal stability (appearance of coagulum).

\section{Molecular Weight Parameters}

The presence of RS (STPO or HTPO) decreases final molecular weights and the decrease is more pronounced with STPO (Table I ). A larger fraction of hydrophilic (mobile) HTPO is consumed by reaction with primary radicals at the beginning of polymerization and therefore the growth of macroradicals at medium or high conversion nearly proceeds under inhibitor-free conditions. This results from the presence of primary radicals (desorbed from the microdroplets) in the aqueous phase. The retarded transfer of STPO (hydrophobe) from the monomer droplets to the reaction loci continuously deactivates growing radicals. Under such conditions, polymer or particle growth is a complex function of particle size, level of hydrophobe in the microdroplet, monomer concentration at the reaction loci, etc. The presence of hydrophobic oligomers in the microdroplets decreases diffusion of monomer to the reaction loci and so the rate of polymerization as well. The addition of inorganic salts (STPO/inorganic salt), slightly increases molecular weight. This may result from partial deactivation of RS by inorganic salt. Some inorganic salts and organic additives act as radical scavengers. This is a well-known reaction of KJ with peroxide (radicals). ${ }^{31}$

This observation reveals the complex nature of microemulsion polymerization initiated by the oil-soluble initiator. The final conclusion about the history and fate of initiating radicals formed in such systems is still open to theoretical and experimental verification.

\section{CONCLUSION}

The addition of STPO and HTPO decreases the rate of polymerization and molecular weight of polymer and the decrease is more pronounced with STPO. The decrease in rate was attributed to reaction of primary or growing radicals with stable radicals. Initiating radicals derived from DBP and formed via chain transfer events are present in microdroplets and aqueous phase. The formation of a more compact structure at the droplet surface by the inorganic salts, organic additives and STPO disfavors the radical entry rate and the exit of radicals from the cage (droplet clusters). A barrier to entering radicals (depressed radical entry) increases the overall activation energy of polymerization. Initiating radicals are formed by 1) decomposition of DBP dissolved in the oil-phase (microdroplets) and subsequent desorption of one of primary radicals to the aqueous phase, 2) decomposition of DBP dissolved in water and 3) exit of primary radicals from the radical pairs (a cage) via a decay of droplet clusters into the individual droplets. The decrease of the particle size in the presence of RS is attributed to shortstopping events and decreased diffusional degradation of microdroplets. The shift of maximum rate of polymerization to lower conversion results from increased number of polymer particles, smaller droplet core and faster consumption of the droplet (monomer) core.

Acknowledgment. This research was supported by the Slovak Grand Agency (VEGA) through the grant number 2/5005/98. The author thanks Mrs. O. Jurikova for assistance in the experiments.

\section{REFERENCES}

1. I. Capek and P. Potisk, Eur. Polym. J., 31, 1269 (1995).

2. P. Potisk and I. Capek, Angew. Makromol. Chem., 222, 125(1994).

3. I. Capek, Polym. Int ., 40, 41 (1996).

4. J. Bartoň, and I. Capek, "Radical Polymerization in Disperse Systems," Ellis Howard, Chichester, U.K., and Veda, Bratislava, 1994.

5. K. Nomura and K. Fujita, Makromol . Chem. Rapid Commun., 10, 581 (1989).

6. J. M. Asua, V. S. Lodriguetz, E. D. Sudol, and M. S. El-Aasser, J. Polym. Sci., Polym. Chem. Ed., 27, 3569 (1989).

7. J. A. Alducin, J. Forcada, and J. M. Asua, Macromolecules, 27, 2256 (1994).

8. S. Holdcroft and J. E. Guillet, J. Polym. Sci., Part A : Polym. Chem., 28, 1823 (1990)

9. D. R. Kim and D. H. Napper, Macromol. Rapid Commun., 17, 845 (1996).

10. J. S. Guo, E. D. Sudol, J. W. Vanderhoff, and M. S. Elaasser., J. Polym. Sci., Part A : Polym. Chem., 30, 691 (1992), ibid., 30, 703 (1992).

11. I. Capek and J. Barton̆, Makromol. Chem., 186, 1297 (1985).

12. I. Capek and J. Bartoň, Chem. Papers, 40, 45 (1986).

13. I. Capek, Makromol. Chem., 190, 789 (1989).

14. P. Potisk, V. Vašková, I. Capek, and M. Klimová, Angew. Makromol. Chem., 236, 43 (1996).

15. L. T. Pearson, P. E. J. Louis, R. G. Gilbert, and D. H. Napper, J. Polym. Sci., Polym. Chem. Ed., 29, 515 (1991).

16. J. Reimers and F. J. Shork, J. Appl. Polym. Sci., 59, 1833 (1996).

17. T. Tanrisever, O. Okay, and I. C. Sonmezoglu, J. Appl. Polym. Sci., 61, 485 (1996).

18. J. L. Reimers and F. J. Schork, J. Appl. Polym. Sci., 60, 251 (1996).

19. J. Brandrup and E. H. Immergut, Ed, "Polymer Handbook," 3rd Ed, John Wiley and Sons, New York, N.Y., 1989.

20. P. Paglioni, S. Ferroni, G. Martini, and M. F. Ottawiani, J. Phys. 
Chem., 88, 5107 (1984).

21. A. Barelli and H. F. Eicke, Langmuir, 2, 780 (1986).

22. Y. T. Choi, M. S. El-Aasser, E. D. Sudol, and J. W. Vanderhof, $J$. Polym. Sci., Polym. Chem.Ed., 23, 2973 (1985).

23. V. Vašková, Z. Hlouskova, J. Bartoñ, and V. Juranicǒvá, Makromol. Chem., 193, 627 (1992).

24. C. J. Hawker, Acc. Chem. Res., 30, 373 (1997),

25. L. M. Gan, C. H. Chew, S. C. Ng, and S. E. Loh, Langmuir, 9, 2799 (1993).

26. M. T. Carver, E. Hirsch, J. C. Wittmann, R. M. Fitch, and F.Candau, J. Phys. Chem., 93, 4867 (1989).

27. N. Kamenka, I. Burgaud, C. Treiner, and R. Zana, Langmuir, 10, 3455 (1994).

28. R. Nagarajan, Colloid Surf., 13, 1 (1985).

29. M. Valiente and E. Rodenas, Colloid Polym. Sci., 271, 494 (1993).

30. M. E. Diaz Garcia, and A. Sanz-Medel, Talanta, 33, 255 (1986).

31. J. Barton and E. Borsig, "Complexes in Free-Radical Polymeriza- tion," Elsevier, Amsterdam, 1988.

32. S. Brunetti, D. Roux, A. M. Bellocq, G. Forche, and P. Bothorel, $J$. Phys. Chem., 87, 1028 (1983).

33. J. Bartoň, I. Capek, M. Arnold, and M. Ratzsch, Makromol. Chem., 181, 241 (1980).

34. S. Gutfelt, J. Kizling, and K. Holmberg, Colloids and Surfaces A: Physicochem. Eng. Aspects 128, 265 (1997).

35. M. Bourrel and R. D. Schechter, "Microemulsions and Related Systems," Marcel Dekker, Inc., New York, N.Y., 1988.

36. R. Zana, W. Binana-Limbele, N. Kamenka, and B. Lindman, $J$. Phys. Chem., 96, 5461 (1992).

37. I. Piirma and S. R. Chen, J. Colloid Interface Sci., 74, 90 (1980).

38. J. M. Peacock and E. Matijevic, J. Colloid Interface Sci., 77, 548 (1980).

39. C. M. Miller, E. D. Sudol, C. A. Silebi, and M. S. El-Aasser, Macromolecules, 28, 2754 (1995).

40. P. Paton and F. I. Talens, J. Surfactants Detergents, 1, 1 (1998). 